\title{
Low-tube-voltage selection for triple-rule-out CTA: relation to patient size
}

\author{
Richard A. P. Takx ${ }^{1}$ - Radko Krissak ${ }^{2,3}$ - Christian Fink ${ }^{2,4}$ - Valentin Bachmann ${ }^{2}$. \\ Thomas Henzler $^{2} \cdot$ Mathias Meyer $^{2} \cdot$ John W. Nance ${ }^{5} \cdot$ Stefan O. Schoenberg ${ }^{2}$ • \\ Paul Apfaltrer ${ }^{2,6}$
}

Received: 5 June 2016 / Revised: 18 August 2016 / Accepted: 7 September 2016 / Published online: 29 September 2016

(C) The Author(s) 2016. This article is published with open access at Springerlink.com

\begin{abstract}
Objectives To investigate the relationship between image quality and patient size at 100 kilovoltage $(\mathrm{kV})$ compared to $120 \mathrm{kV}$ ECG-gated Triple-Rule-Out CT angiography (TRO-CTA). Methods We retrospectively included 73 patients (age $64 \pm$ 14 years) who underwent retrospective ECG-gated chest CTA. 40 patients were scanned with $100 \mathrm{kV}$ while 33 patients with $120 \mathrm{kV}$. Body mass index (BMI), patients' chest circumference (PC) and thoracic surface area (TSA) were recorded. Quantitative image quality was assessed as vascular attenuation in the ascending aorta (AA), pulmonary trunk (PA) and left coronary artery (LCA) and the signal-to-noise ratio (SNR) in the AA.

Results There was no significant difference in BMI $(26.0 \pm 4.6$ vs. $\left.28.0 \pm 6.7 \mathrm{~kg} / \mathrm{m}^{2}\right), \mathrm{PC}\left(103 \pm 7\right.$ vs. $\left.104 \pm 10 \mathrm{~cm}^{2}\right)$ and TSA $\left(92 \pm 15\right.$ vs. $\left.91 \pm 19 \mathrm{~cm}^{2}\right)$ between $100 \mathrm{kV}$ and $120 \mathrm{kV}$ group. Mean vascular attenuation was significantly higher in
\end{abstract}

Richard A. P. Takx

r.a.p.takx@umcutrecht.nl

1 Department of Radiology, University Medical Center Utrecht, Heidelberglaan 100, P.O. Box 85500, 3584

CX Utrecht, The Netherlands

2 Institute of Clinical Radiology and Nuclear Medicine, University Medical Center Mannheim, Medical Faculty Mannheim Heidelberg University, Heidelberg, Germany

3 Department of Diagnostic and Interventional Radiology, Hufeland Klinikum GmbH, Bad Langensalza, Germany

4 Department of Radiology, General Hospital Celle, Celle, Germany

5 Division of Cardiovascular Imaging, Department of Radiology and Radiological Science, Medical University of South Carolina, Charleston, SC, USA

6 Department of Biomedical Imaging and Image-guided Therapy, Medical University of Vienna, Vienna, Austria the $100 \mathrm{kV}$ compared to the $120 \mathrm{kV}$ group (AA 438 vs. 354 HU, PA 460 vs. 349 HU, LCA 370 vs. 299 HU all $p<0.001$ ). SNR was not significantly different, even after adjusting for patient size. Radiation dose was significantly lower in the $100 \mathrm{kV}$ group $(10.7 \pm 4.1$ vs. $20.7 \pm 10.7 \mathrm{mSv} ; p<0.001)$. Conclusions $100 \mathrm{kV}$ TRO-CTA is feasible in normal-tooverweight patients while maintaining image quality and achieving substantial dose reduction.

Key Points

- $100 \mathrm{kV}$ protocols result in a significantly lower radiation dose.

- Mean vascular attenuation is significantly higher using $100 \mathrm{kV}$.

- SNR and CNR are not significantly different between $100 \mathrm{kV}$ and $120 \mathrm{kV}$.

- $100 \mathrm{kV} \mathrm{CTA}$ is feasible regardless of patient size while maintaining image quality.

Keywords Multidetector computed tomography $\cdot$ Radiation dosage $\cdot$ Image enhancement $\cdot$ Body mass index $\cdot$ Chest pain

$\begin{array}{ll}\text { Abbreviations } \\ \text { BMI } & \text { body mass index } \\ \text { CTA } & \text { computed tomography angiography } \\ \text { CTDI }_{\text {vol }} & \text { volume CT dose index } \\ \text { DLP } & \text { dose length product } \\ \text { kV } & \text { kilovoltage } \\ \text { PC } & \text { patients' chest circumference } \\ \text { TRO } & \text { triple rule out } \\ \text { TSA } & \text { thoracic surface area }\end{array}$

\section{Introduction}

ECG-gated computed tomography angiography (CTA) of the chest has been shown to be a reliable and comprehensive non- 
invasive imaging test to exclude acute coronary syndrome, aortic dissection and pulmonary embolism in patients presenting to the emergency department with acute chest pain (i.e., Triple Rule Out (TRO)-CTA) [1-9].

However, the potential harm of radiation exposure is of concern, as it has been postulated to be associated with a non-negligible lifetime attributable risk of cancer [10]. This risk varies noticeably and is substantially greater for females, younger patients, and for combined cardiac and aortic scans [10]. Previous studies demonstrated that lowering the kilovoltage $(\mathrm{kV})$ setting from standard $120 \mathrm{kV}$ to $100 \mathrm{kV}$ allows a significant reduction of radiation exposure independent from the CT scanner hardware [11-14]. Radiation dose reduction through $\mathrm{kV}$ adjustment, however, may adversely affect image quality due to the inverse relationship between radiation exposure and image noise [15]. Empiric selection criteria for application of reduced peak tube potential include markers of body size (e.g., body mass index [BMI]); however, body size selection criteria are likely crude given interpatient differences in weight distribution and body composition [15]. Patients' chest area and BMI have been found to frequently be discordant, which can lead to over radiating patients when using BMI to select tube potential [16]. However, there remains hesitation among clinicians to lower $\mathrm{kV}$ out of fear for not achieving adequate image quality for diagnosis. The hypothesis of this study was that $100 \mathrm{kV}$ CTA is feasible in normal-to-overweight patients while maintaining appropriate diagnostic image quality. Accordingly, we investigated the relationship between image quality and patient size in patients undergoing 100 kV vs. 120 kV ECG-gated TRO-CTA.

\section{Materials and methods}

\section{Patient population}

Seventy-three consecutive patients (mean age $64 \pm 13$, range 25-83 years) were retrospectively enrolled. All patients underwent TRO-CTA for acute chest pain evaluation referred by our chest pain unit between October 2009 and January 2011. The $100 \mathrm{kV}$ scanning was performed at the discretion of the attending radiologist. In our institution, TRO-CTA is used to assess patients with acute chest pain for pulmonary embolism, acute aortic syndrome, acute coronary syndrome and other thoracic pathologic conditions with a single examination.

\section{CT technique}

All imaging exams were conducted on a 64-channel dualsource CT scanner (Somatom Definition, Siemens Healthcare, Forchheim, Germany). Retrospectively ECGgated spiral CTA of the heart was acquired from the level of the carina. Then, $120 \mathrm{ml}$ of iodine contrast (Imeron 400, Bracco Imaging S.p.A., Milan, Italy) was injected using a power injector with an injection rate of $4 \mathrm{ml} / \mathrm{s}$ and followed by a saline chaser of $40 \mathrm{ml}$. Patients were instructed to hold their breath at a mid-inspiratory position. The cranio-caudal acquisition was started using automatic bolus triggering at $2 \mathrm{sec}$ intervals in the ascending aorta (trigger level $100 \mathrm{HU}$ ) with a delay of $5 \mathrm{sec}$. The following additional scan parameters were applied for the TRO-CTA: detector collimation $2 \times 32 \times$ $0.6 \mathrm{~mm}$, slice acquisition $2 \times 64 \times 0.6 \mathrm{~mm}$ using $\mathrm{z}$-flying focal spot, gantry rotation time $330 \mathrm{~ms}$, tube current time product $320 \mathrm{mAs}$, and the pitch automatically adapted to the patient's heart rate using a reference value of 0.28. Automatic tube current modulation (CARE Dose 4D) and automatic ECG-pulsing for radiation dose reduction were applied. For data reconstruction, axial images of the thoracic scan range were reconstructed with $1.0 \mathrm{~mm}$ slice thickness and $0.8 \mathrm{~mm}$ increment using a B30f soft tissue kernel. In addition, the heart was reconstructed with a slice thickness of $0.6 \mathrm{~mm}$ and $0.5 \mathrm{~mm}$ increment using a B26f soft tissue kernel in the optimal diastolic and systolic phase as determined by the CT software. Dose length product (DLP) and volume $\mathrm{CT}$ dose index $\left(\mathrm{CTDI}_{\mathrm{vol}}\right)$ were collected. The effective dose was calculated using a conversion coefficient of $0.017 \mathrm{mSv} \mathrm{mGy}^{-1} \mathrm{~cm}^{-1}$ [17].

\section{Image analysis}

The quantitative measurements were performed on the axial images with $1 \mathrm{~mm}$ slice thickness by one experienced reviewer (radiologist with 5 years of experience in cardiothoracic imaging). A circular region of interest (ROI) was placed in the following anatomical regions: main pulmonary artery, ascending aorta, left ventricle, right ventricle, left main coronary artery, and epicardial fat. Calcifications and plaques within the ROIs were avoided. Mean attenuation values were recorded in Hounsfield Units (HU). The signal-to-noise ratio (SNR) was defined as the ratio of the mean attenuation in the ascending aorta to the standard deviation of this ROI. Contrast-to-noise ratio (CNR) was defined as the ratio of the mean attenuation in the ascending aorta minus mean attenuation of epicardial fat to the standard deviation of the attenuation value in the ascending aorta. Patient size was measured as BMI, patients' chest circumference (PC) at the level of the origin of the left main coronary and thoracic surface area (TSA), defined as the product of dorso-ventral and latero-lateral diameters of the chest at the same level as above (Fig. 1).

\section{Statistical analysis}

Continuous variables were listed as mean plus standard deviation (SD). Student's t-test was used to determine statistical significant differences in mean values. Linear regression was 
Fig. 1 Example image of measurements (a) ROI in ascending aorta and pulmonary artery. b Left main coronary artery and epicardial fat. $\mathbf{c}$ left and right ventricle. d anthropomorphic measures at level of the origin of the left main coronary artery
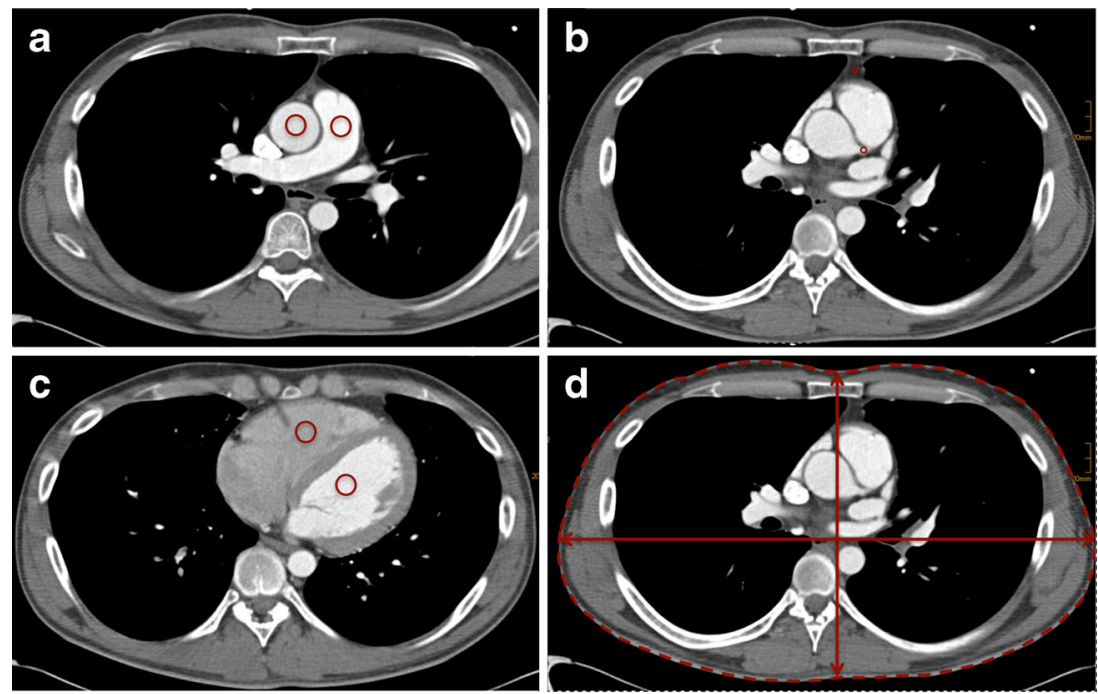

applied to generate adjusted $p$-values. Correlations were calculated using Pearson correlation coefficient or Spearman's rank. Discrete variables were evaluated using the Fisher's exact test. A $p$-value smaller than 0.05 was used to indicate statistical significance. Analyses were performed using SPSS (IBM Corp, Version 23.0. Armonk, NY, USA).

\section{Results}

\section{Baseline characteristics}

There was no significant difference in BMI between the $100 \mathrm{kV}$ and $120 \mathrm{kV}$ group $\left(26.0 \pm 4.6\right.$ vs. $28.0 \pm 6.7 \mathrm{~kg} / \mathrm{m}^{2}$; $p=0.138)$, PC (103 \pm 7 vs. $\left.104 \pm 10 \mathrm{~cm}^{2} ; p=0.737\right)$ or TSA $\left(92.4 \pm 15.2\right.$ vs. $\left.90.9 \pm 19.2 \mathrm{~cm}^{2} ; p=0.815\right)$. Both groups showed similar age $(65 \pm 12$ vs. $63 \pm 16$ years; $p=0.521)$ and similar proportion of males ( 27 vs. 20 males, $p=0.540$ ). Detailed characteristics of the study population are listed in Table 1. The correlation between BMI and TSA/PC was moderate while the correlation between TSA and PC was high (Fig. 2).

Table 1 Patient characteristics and radiation dose

\begin{tabular}{lllr}
\hline & $100 \mathrm{kV}(n=40)$ & $120 \mathrm{kV}(n=33)$ & $p$-value \\
\hline BMI $\left(\mathrm{kg} / \mathrm{m}^{2}\right)$ & $26.0 \pm 4.6$ & $28.0 \pm 6.7$ & 0.138 \\
$<25$ & 19 & 14 & \\
$25.1-30$ & 17 & 11 & \\
$>30.1$ & 4 & 8 & $<0.001$ \\
CTDI (mGy) & $19.2 \pm 6.9$ & $41.3 \pm 21.5$ & 0.044 \\
mAs & $162.4 \pm 55.6$ & $192.9 \pm 70.8$ & $<0.001$ \\
DLP (mGy cm) & $630 \pm 240$ & $1215 \pm 626$ & \\
\hline
\end{tabular}

\section{Image quality}

A radiologist (5 years of experience) graded all studies as of diagnostic quality. The average attenuation of contrast medium in the low-dose group was significantly higher at all vascular levels (all $p<0.05$; Table 2; Fig. 3). There was no significant difference in the mean attenuation values of epicardial fat between the groups $(p=0.40)$. Image noise in the main pulmonary artery and left main coronary artery was higher in the low-voltage group $(p<0.05)$. Image noise in the right ventricle and epicardial fat showed no significant difference as presented in Table 2 .

The difference in the calculated SNR and CNR between the two groups was not statistically significant $(p=0.46$ and $p=0.62$ ), which was maintained after adjusting for body size measures.

\section{Radiation dose}

Mean $\mathrm{CTDI}_{\mathrm{vol}}$ in the $100 \mathrm{kV}$ group was $19.2 \pm 6.9 \mathrm{mGy}$ and was significantly lower compared to the $120 \mathrm{kV}$ group $(41.3 \pm 21.5 \mathrm{mGy}, p<0.001)$. Mean DLP in the $100 \mathrm{kV}$ group was $630 \pm 240 \mathrm{mGy} \mathrm{cm}$ and was significantly lower in comparison with the $120 \mathrm{kV}$ group (1215 $\pm 626 \mathrm{mGy} \mathrm{cm}, p<0.001)$. The mean estimated effective radiation dose for the $100 \mathrm{kV}$ group was $10.7 \pm 4.1 \mathrm{mSv}$ and $20.7 \pm 10.7 \mathrm{mSv}(p<0.001)$, corresponding to a $55 \%$ reduction in radiation exposure by lowering tube voltage to $100 \mathrm{kV}$. Radiation dose results are summarized in Table 1. A moderate correlation was found between BMI and effective radiation dose for both the $100 \mathrm{kV}$ group and the $120 \mathrm{kV}$ group $(r=0.326, p=0.043$ and $r=0.357$, $p=0.041$, respectively). 

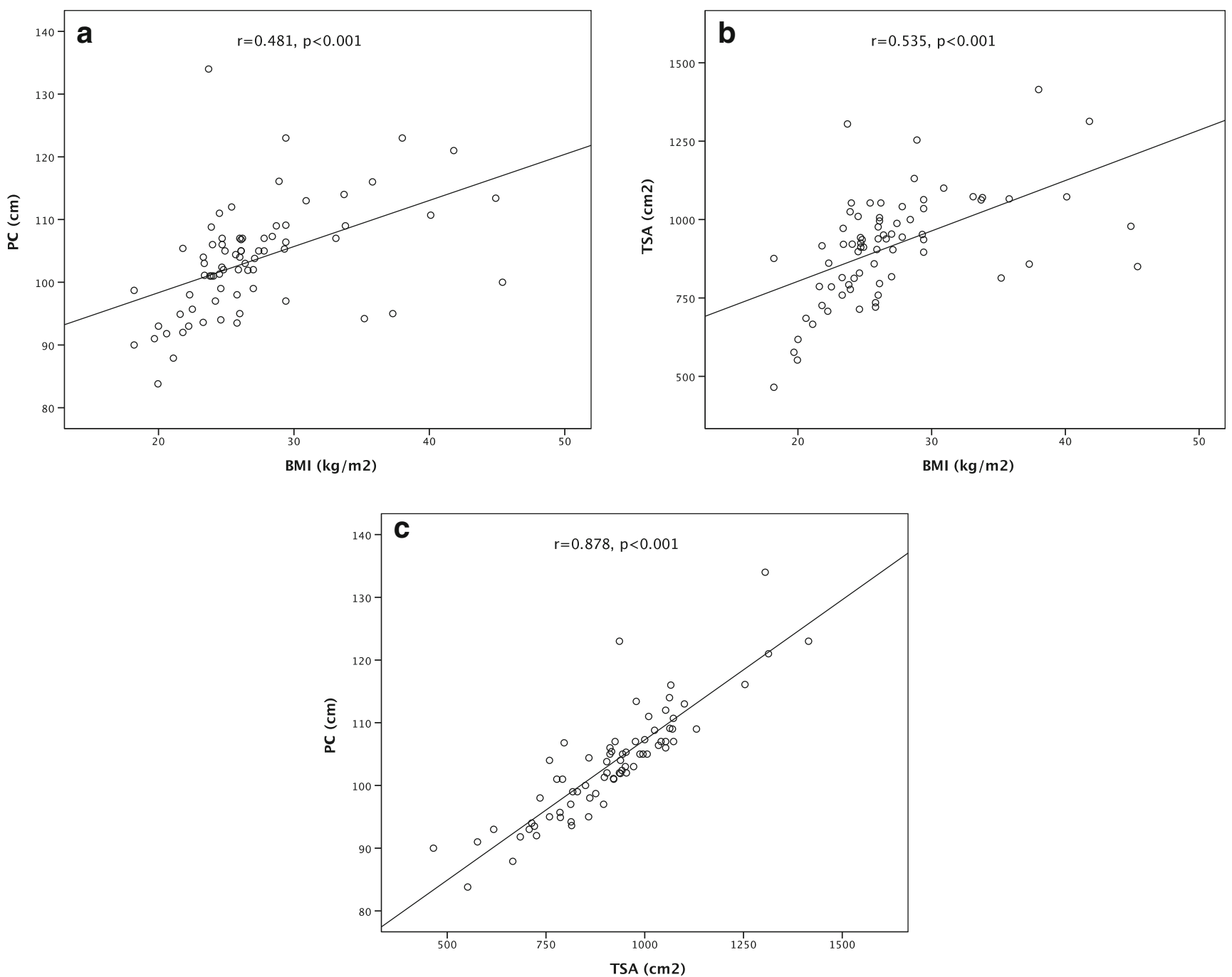

Fig. 2 Correlation plot between the various anthropomorphic measures. a PC and BMI, b TSA and BMI, and $\mathbf{c}$ PC and TSA

\section{Discussion}

Our results show that $100 \mathrm{kV}$ TRO-CTA of the chest can be performed with appropriate diagnostic image quality and substantial dose reduction in our patient population, which included predominantly normal-to-overweight individuals. Even after adjusting for measures of patient size, no significant differences were found in image quality between $100 \mathrm{kV}$ and $120 \mathrm{kV}$. Reducing tube voltage to $100 \mathrm{kV}$ results in an approximately $55 \%$ lower effective radiation dose compared to a $120 \mathrm{kV}$ TRO-CTA protocol performed while maintaining diagnostic image quality. Similar reductions were reported by Feuchtner et al. [12], where a radiation dose reduction of $47 \%$ was achieved using $100 \mathrm{kV}$.

The mean attenuation values of the pulmonary artery, the ascending aorta, the left ventricle, the right ventricle and the left main coronary artery were significantly higher at $100 \mathrm{kV}$, which is in line with published data on low $\mathrm{kV}$ imaging $[18,19]$.
This observation can be explained by the photoelectric effect, which results in increased photon attenuation as tube voltages approach the k-edge of a given element; in case of iodine-based contrast materials, vascular attenuation is expected to increase with decreasing tube voltages to approximately $33 \mathrm{kV}$ (the approximate k-edge of iodine) [20, 21]. The photoelectric effect is the dominant mechanism of photon attenuation in materials containing high-atomic number elements (e.g., iodinated contrast material). In contrast, Compton scatter is the dominant interaction in tissues lacking high-atomic number elements (e.g., soft tissue and fat). Accordingly, no significant difference in mean attenuation of the (fatty) tissue adjacent to the left coronary artery was observed [22]. A disadvantage of lower tube voltage (and the major reason for diagnostic tube voltage ranges used in current clinical imaging) is the concomitant increase in image noise [20]. While the noise level for the $100 \mathrm{kV}$ CTA protocol was found to be higher in the current study, as expected, all imaging studies were considered of diagnostic quality, which 
Table 2 Mean attenuation and noise

\begin{tabular}{|c|c|c|c|c|c|c|}
\hline & $100 \mathrm{kV}(n=40)$ & $120 \mathrm{kV}(n=33)$ & $p$-value & $\begin{array}{l}\mathrm{p} \text {-value adjusted } \\
\text { for BMI }\end{array}$ & $\begin{array}{l}\mathrm{p} \text {-value adjusted } \\
\text { for PC }\end{array}$ & $\begin{array}{l}\mathrm{p} \text {-value adjusted } \\
\text { for TSA }\end{array}$ \\
\hline \multicolumn{7}{|l|}{ Attenuation } \\
\hline Pulmonary artery & $459.8 \pm 107.2$ & $348.6 \pm 85.3$ & $<0.001$ & $<0.001$ & $<0.001$ & $<0.001$ \\
\hline Ascending Aorta & $435.7 \pm 102.4$ & $353.6 \pm 66.9$ & $<0.001$ & $<0.001$ & $<0.001$ & $<0.001$ \\
\hline Left ventricle & $407.9 \pm 97.6$ & $326.2 \pm 68.9$ & $<0.001$ & $<0.001$ & $<0.001$ & $<0.001$ \\
\hline Right ventricle & $405.5 \pm 135.3$ & $335.3 \pm 118.9$ & 0.024 & 0.040 & 0.029 & 0.021 \\
\hline Left coronary artery & $369.5 \pm 117.1$ & $299.4 \pm 101.3$ & 0.009 & 0.015 & 0.011 & 0.009 \\
\hline Epicardial fat & $99.8 \pm 25.5$ & $93.9 \pm 25.6$ & 0.335 & 0.404 & 0.367 & 0.336 \\
\hline \multicolumn{7}{|l|}{ Noise } \\
\hline Pulmonary artery & $30.3 \pm 10.9$ & $22.3 \pm 8.5$ & 0.001 & $<0.001$ & $<0.001$ & 0.001 \\
\hline Ascending Aorta & $27.4 \pm 9.2$ & $22.3 \pm 9.1$ & 0.057 & 0.061 & 0.049 & 0.060 \\
\hline Left ventricle & $36.6 \pm 15.7$ & $31.9 \pm 22.9$ & 0.302 & 0.188 & 0.212 & 0.269 \\
\hline Right ventricle & $46.3 \pm 21.7$ & $40.8 \pm 24.7$ & 0.315 & 0.231 & 0.258 & 0.303 \\
\hline Left main coronary artery & $67.9 \pm 28.5$ & $48.3 \pm 21.9$ & 0.002 & 0.001 & 0.002 & 0.002 \\
\hline Epicardial fat & $33.9 \pm 14.3$ & $32.0 \pm 20.0$ & 0.649 & 0.657 & 0.594 & 0.645 \\
\hline CNR aorta & $12.6 \pm 4.7$ & $13.8 \pm 8.9$ & 0.463 & 0.419 & 0.391 & 0.453 \\
\hline SNR aorta & $16.9 \pm 6.9$ & $17.7 \pm 6.9$ & 0.623 & 0.460 & 0.529 & 0.666 \\
\hline
\end{tabular}

was supported by non-significant differences in SNR and CNR between both protocols. In a prior study on $100 \mathrm{kV}$ imaging by Krissak et al. [22] the feasibility of TRO-CTA with $100 \mathrm{kV}$ was demonstrated in non-obese patients. In this current study we extend the use of $100 \mathrm{kV}$ imaging to normal-to-overweight patients, which was maintained even after adjusting for measures of patient size.

Despite growing evidence of non-inferior image quality using low $\mathrm{kV}$ protocols in nonobese patients [11, 22], data
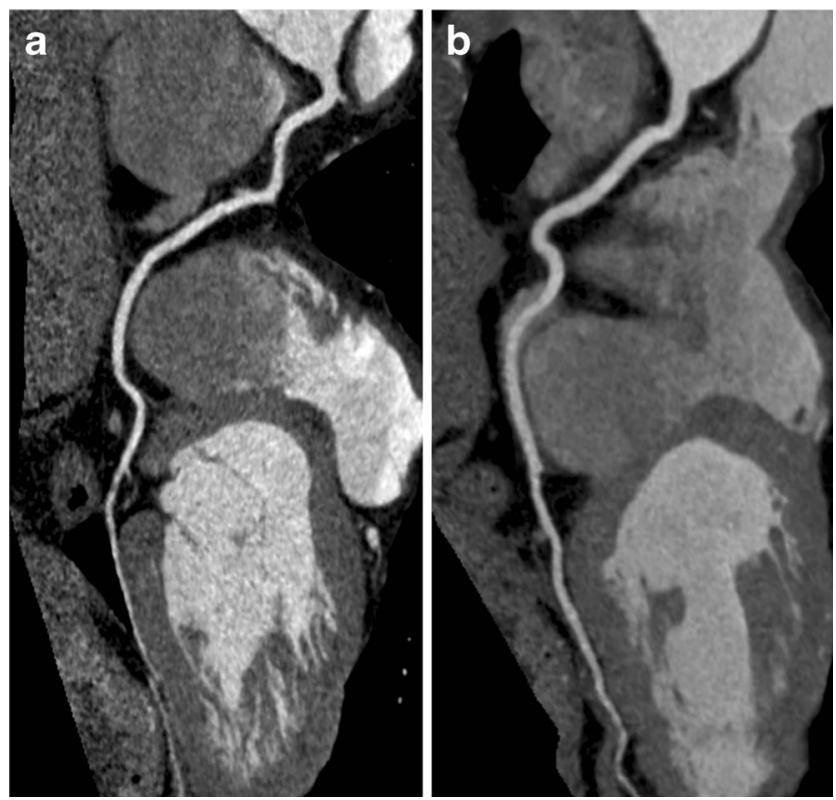

Fig. 3 The right coronary artery in patients of similar BMI scanned with Triple-Rule-Out CT angiography at $100 \mathrm{kV}$ (a) and $120 \mathrm{kV}$ (b) regarding the impact of body habitus, i.e., chest dimensions, PC, and TSA has been lacking. This is of particular concern, as a recent anthropomorphic phantom study found that patients' chest area and BMI classes were frequently discordant, which could potentially lead to over-radiating patients when using BMI to select the tube voltage [16]. BMI, while an imperfect but convenient proxy for human body fat, does not reliably represent the body shape, especially in patients with central obesity; this can become particularly troublesome in TRO-CTA, where the scan field is limited to the chest. Conversely, a large-chested woman probably requires more radiation than a small-chested man with a similar BMI [23]. Thus, the findings of our study extend prior data, establishing radiation dose reduction and maintaining image quality even after adjusting for patient size.

Our study is limited by its retrospective nature. Second, we only had a small percentage of subjects with a BMI above 30 , which may hamper the generalizability of the results to morbidly obese individuals. Finally, in this study we did not evaluate the effect of iterative image reconstruction techniques, which would reduce image noise $[24,25]$.

In conclusion, $100 \mathrm{kV}$ TRO-CTA can be performed in normal-to-overweight patients, while maintaining image quality and achieving substantial dose reductions.

Acknowledgments The scientific guarantor of this publication is Richard A.P. Takx. The authors of this manuscript declare relationships with the following companies: The authors of this manuscript declare no relationships with any companies, whose products or services may be related to the subject matter of the article. 
The authors state that this work has not received any funding. One of the authors has significant statistical expertise. Institutional Review Board approval was obtained. Written informed consent was waived by the Institutional Review Board. Methodology: retrospective, cross sectional study, performed at one institution.

Open Access This article is distributed under the terms of the Creative Commons Attribution 4.0 International License (http:// creativecommons.org/licenses/by/4.0/), which permits unrestricted use, distribution, and reproduction in any medium, provided you give appropriate credit to the original author(s) and the source, provide a link to the Creative Commons license, and indicate if changes were made.

\section{References}

1. Halpern EJ (2009) Triple-rule-out CT angiography for evaluation of acute chest pain and possible acute coronary syndrome. Radiology 252:332-345

2. Frauenfelder T, Appenzeller P, Karlo C et al (2009) Triple rule-out $\mathrm{CT}$ in the emergency department: protocols and spectrum of imaging findings. Eur Radiol 19:789-799

3. Christiaens L, Duchat F, Boudiaf M et al (2012) Impact of 64-slice coronary $\mathrm{CT}$ on the management of patients presenting with acute chest pain: results of a prospective two-centre study. Eur Radiol 22: $1050-1058$

4. Schertler T, Scheffel H, Frauenfelder T et al (2007) Dual-source computed tomography in patients with acute chest pain: feasibility and image quality. Eur Radiol 17:3179-3188

5. Zhang LJ, Lu GM, Meinel FG, McQuiston AD, Ravenel JG, Schoepf UJ (2015) Computed tomography of acute pulmonary embolism: state-of-the-art. Eur Radiol 25:2547-2557

6. Durmus T, Rogalla P, Lembcke A, Muhler MR, Hamm B, Hein PA (2011) Low-dose triple-rule-out using 320-row-detector volume MDCT-less contrast medium and lower radiation exposure. Eur Radiol 21:1416-1423

7. Rist C, Johnson TR, Becker CR, Reiser MF, Nikolaou K (2007) New applications for noninvasive cardiac imaging: dual-source computed tomography. Eur Radiol 17:F16-F25

8. Wnorowski AM, Halpern EJ (2016) Diagnostic yield of triple-rule-Out CT in an emergency setting. AJR Am J Roentgenol 207:295-301

9. Kligerman SJ, White CS (2014) Image quality and feasibility of an ultralow-dose high-pitch helical triple-rule-out computed tomography angiography acquired in the caudocranial direction. J Thorac Imaging 29:50-59

10. Einstein AJ, Henzlova MJ, Rajagopalan S (2007) Estimating risk of cancer associated with radiation exposure from 64-slice computed tomography coronary angiography. JAMA 298:317-323

11. Bischoff B, Hein F, Meyer T et al (2009) Impact of a reduced tube voltage on CT angiography and radiation dose: results of the PROTECTION I study. JACC Cardiovasc Imaging 2:940-946
12. Feuchtner GM, Jodocy D, Klauser A et al (2010) Radiation dose reduction by using $100-\mathrm{kV}$ tube voltage in cardiac 64-slice computed tomography: a comparative study. Eur J Radiol 75:e51-e56

13. Fink C, Krissak R, Henzler T et al (2011) Radiation dose at coronary $\mathrm{CT}$ angiography: second-generation dual-source $\mathrm{CT}$ versus single-source 64-MDCT and first-generation dual-source CT. AJR Am J Roentgenol 196:W550-W557

14. Leschka S, Stolzmann P, Schmid FT et al (2008) Low kilovoltage cardiac dual-source CT: attenuation, noise, and radiation dose. Eur Radiol 18:1809-1817

15. Ghafourian K, Younes D, Simprini LA, Weigold WG, Weissman G, Taylor AJ (2012) Scout view X-ray attenuation versus weightbased selection of reduced peak tube voltage in cardiac CT angiography. JACC Cardiovasc Imaging 5:589-595

16. Ghoshhajra BB, Engel LC, Major GP et al (2011) Direct chest area measurement: a potential anthropometric replacement for BMI to inform cardiac CT dose parameters? J Cardiovasc Comput Tomogr 5:240-246

17. Commission E (1999) European guidelines on quality criteria for computed tomography. Brussels, Belgium Report EUR 16262 EN

18. Kalva SP, Sahani DV, Hahn PF, Saini S (2006) Using the K-edge to improve contrast conspicuity and to lower radiation dose with a 16MDCT: a phantom and human study. J Comput Assist Tomogr 30: 391-397

19. Kidoh M, Nakaura T, Nakamura $\mathrm{S}$ et al (2014) Contrast material and radiation dose reduction strategy for triple-rule-out cardiac CT angiography: feasibility study of non-ECG-gated low $\mathrm{kVp}$ scan of the whole chest following coronary CT angiography. Acta Radiol 55:1186-1196

20. Huda W, Scalzetti EM, Levin G (2000) Technique factors and image quality as functions of patient weight at abdominal CT. Radiology 217:430-435

21. Sigal-Cinqualbre AB, Hennequin R, Abada HT, Chen X, Paul JF (2004) Low-kilovoltage multi-detector row chest CT in adults: feasibility and effect on image quality and iodine dose. Radiology 231: $169-174$

22. Krissak R, Henzler T, Prechel A et al (2010) Triple-rule-out dual-source CT angiography of patients with acute chest pain: Dose reduction potential of $100 \mathrm{kV}$ scanning. Eur J Radiol. doi:10.1016/j.ejrad.2010.11.021

23. Li JL, Huang MP, Liang $\mathrm{CH}$ et al (2012) Individualized radiation dose control in 256-slice CT coronary angiography (CTCA) in retrospective ECG-triggered helical scans: Using a measure of body size to adjust tube current selection. Eur J Radiol. doi:10.1016/j. ejrad.2012.04.017

24. Moscariello A, Takx RA, Schoepf UJ et al (2011) Coronary CT angiography: image quality, diagnostic accuracy, and potential for radiation dose reduction using a novel iterative image reconstruction technique-comparison with traditional filtered back projection. Eur Radiol 21:2130-2138

25. Lu GM, Luo S, Meinel FG et al (2014) High-pitch computed tomography pulmonary angiography with iterative reconstruction at $80 \mathrm{kVp}$ and $20 \mathrm{~mL}$ contrast agent volume. Eur Radiol 24:32603268 